\title{
Creatinine Versus Cystatin C: Differing Estimates of Renal Function in Hospitalized Veterans Receiving Anticoagulants
}

\author{
Christina Hao Wang, MD ${ }^{1,2}$, Anna D. Rubinsky, PhD³, Tracy Minichiello, MD', \\ Michael G. Shlipak, MD, MPH ${ }^{1,2,3,4}$, and Erika Leemann Price, $M D, M^{\prime 1,2}$ \\ 'San Francisco Veterans Affairs Medical Center, San Francisco, CA, USA; '2Department of Medicine, University of California, San Francisco (UCSF), \\ San Francisco, CA, USA; ${ }^{3}$ Kidney Health Research Collaborative, UCSF and SFVAMC, San Francisco, CA, USA; ${ }^{4}$ Department of Epidemiology \& \\ Biostatistics, UCSF, San Francisco, CA, USA.
}

BACKGROUND: Current practice in anticoagulation dosing relies on kidney function estimated by serum creatinine using the Cockcroft-Gault equation. However, creatinine can be unreliable in patients with low or high muscle mass. Cystatin $\mathrm{C}$ provides an alternative estimation of glomerular filtration rate (eGFR) that is independent of muscle.

OBJECTIVE: We compared cystatin C-based eGFR $\left(\mathrm{eGFR}_{\text {cys }}\right)$ with multiple creatinine-based estimates of kidney function in hospitalized patients receiving anticoagulants, to assess for discordant results that could impact medication dosing.

DESIGN: Retrospective chart review of hospitalized patients over 1 year who received non-vitamin $\mathrm{K}$ antagonist anticoagulation, and who had same-day measurements of cystatin $\mathrm{C}$ and creatinine.

PARTICIPANTS: Seventy-five inpatient veterans (median age 68) at the San Francisco VA Medical Center (SFVAMC). MAIN MEASURES: We compared the median difference between eGFR by the Chronic Kidney Disease Epidemiology Collaboration (CKD-EPI) study equation using cystatin $\mathrm{C}\left(\mathrm{eGFR}_{\text {cys }}\right.$ ) and eGFRs using three creatininebased equations: CKD-EPI (eGFR $\mathrm{EPI}_{\mathrm{E}}$ ), Modified Diet in

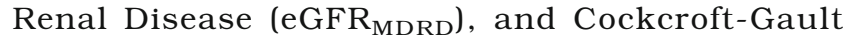
$\left(\mathrm{eGFR}_{\mathrm{CG}}\right)$. We categorized patients into standard KDIGO kidney stages and into drug-dosing categories based on each creatinine equation and calculated proportions of patients reclassified across these categories based on cystatin C.

KEY RESULTS: Cystatin C predicted overall lower eGFR compared to creatinine-based equations, with a median difference of - $7.1(\mathrm{IQR}-17.2,2.6) \mathrm{mL} / \mathrm{min} / 1.73 \mathrm{~m}^{2}$ versus eGFR $\mathrm{EPI},-21.2(\mathrm{IQR}-43.7,-8.1) \mathrm{mL} / \mathrm{min} / 1.73 \mathrm{~m}^{2}$ versus eGFR ${ }_{M D R D}$, and -25.9 (IQR $\left.-46.8,-8.7\right) \mathrm{mL} /$ $\min / 1.73 \mathrm{~m}^{2}$ versus eGFR $\mathrm{CG}$. Thirty-one to $52 \%$ of

Previously presented as an oral presentation: "Discrepancy in estimated GFR based on cystatin $C$ versus creatinine among inpatient veterans anticoagulation" at the California-Hawaii Regional Society for General Internal Medicine (SGIM) meeting, UCLA, Los Angeles, January 21, 2017.

Electronic supplementary material The online version of this article (https://doi.org/10.1007/s11606-018-4461-3) contains supplementary material, which is available to authorized users.

Received May 15, 2017

Revised October 13, 2017

Accepted April 13, 2018

Published online May 31, 2018 patients were reclassified into lower drug-dosing categories using cystatin $\mathrm{C}$ compared to creatinine-based estimates.

CONCLUSIONS: We found substantial discordance in eGFR comparing cystatin $\mathrm{C}$ with creatinine in this group of anticoagulated inpatients. Our sample size was limited and included few women. Further investigation is needed to confirm these findings and evaluate implications for bleeding and other clinical outcomes.

NIH TRIAL REGISTRY NUMBER: Not applicable

KEY WORDS: cystatin C; chronic kidney disease (CKD); anticoagulation; atrial fibrillation; venous thromboembolism.

J Gen Intern Med 33(8):1299-306

DOI: $10.1007 / \mathrm{s} 11606-018-4461-3$

(c) Society of General Internal Medicine (This is a U.S. Government work and not under copyright protection in the US; foreign copyright protection may apply) 2018

\section{INTRODUCTION}

Use of anticoagulation increases with age-related incidence of atrial fibrillation (AF) and venous thromboembolism (VTE) ${ }^{1}$. Several anticoagulants are renally metabolized and require dose adjustment based on kidney function. ${ }^{2,3}$ Inaccurate estimation of kidney function can increase bleeding risk due to supratherapeutic levels or increase risk of recurrent VTE or stroke with sub-therapeutic levels. ${ }^{4-8}$ These risks are especially high for patients with advanced age and frailty, whose renal function may fluctuate and may be particularly difficult to assess. $^{9}$

Creatinine is the endogenous filtration marker most widely used to estimate glomerular filtration rate (eGFR), the accepted metric for kidney function. ${ }^{10}$ Since creatinine generation depends on muscle mass, variations in muscle mass unrelated to kidney function can affect creatinine-based estimates. Multiple equations have been developed to control for these variations. ${ }^{7,9,11}$ Cockcroft-Gault (eGFR ${ }_{\mathrm{CG}}$ ), the first equation developed to estimate creatinine clearance $(\mathrm{CrCl})$ as a proxy for GFR, includes age, sex, and weight. ${ }^{10}$ The newer Modification

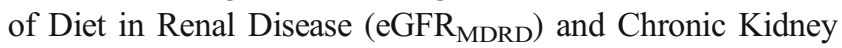
Disease Epidemiology Collaboration $\left(\mathrm{eGFR}_{\mathrm{EPI}}\right)$ equations account for variations in creatinine generation with age, sex, and ethnicity. ${ }^{11}$ While the more recent equations are more 
accurate, challenges remain. Cockcroft-Gault is the least accurate of the creatinine-based equations. It was developed in a cohort of 249 patients without acute or chronic kidney impairment or extremes of body mass, and the equation does not account for changes in creatinine assays since its development. ${ }^{11,12}$ Nonetheless, it remains the basis for most pharmacologic dosing guidelines. ${ }^{2,3}$ All three equations have limited accuracy for patients with extremely low or high body mass, high glomerular filtration rates, advanced age, and non-white/ non-black ethnicities and races. ${ }^{13}$ These equations all rely on serum creatinine, which can fluctuate in acute illness and is often falsely low in chronically ill persons relative to their kidney function. ${ }^{9-11,14}$ Current guidelines recommend against the sole use of creatinine to dose medications with safety concerns. $^{15,16}$

Cystatin C, a $14-\mathrm{kD}$ intracellular protein found in all nucleated cells, is an alternative biomarker for renal function. Unlike creatinine, cystatin $\mathrm{C}$ is neither dependent on muscle mass nor actively secreted by kidney tubules. ${ }^{10,17-23}$ Independent of kidney function, cystatin $\mathrm{C}$ is increased in conditions with high cell turnover, including some malignancies and thyroid disease. Chemotherapy, steroids, and tobacco use may also affect levels. Since cell turnover is proportional to body mass, age, sex, and race are used to correct cystatin C-based estimates of kidney function as with creatininebased equations, but adjustments for these factors are much smaller in cystatin $\mathrm{C}$ equations than in corresponding creatinine equations. ${ }^{11}$ Studies have shown superiority of cystatin $\mathrm{C}$ over creatinine for estimating GFR in ambulatory patients with diabetes, HIV, and early acute kidney injury. Cystatin $\mathrm{C}$ has also shown potential for detecting early renal injury in critically ill patients. ${ }^{24-27} \mathrm{Al}-$ though cystatin $\mathrm{C}$ use is recommended by international guidelines, it has not been well established in clinical practice.

Most studies have evaluated cystatin $\mathrm{C}$ in ambulatory care scenarios, and it has yet to be well studied in the acute inpatient setting. During hospitalization, kidney function is often impaired and creatinine fluctuates. ${ }^{28,29}$ Hospitalized patients, who are typically older and frailer than outpatient populations, are at particular risk for adverse events due to inaccurate estimation of kidney function when taking renally metabolized medications.

We sought to determine how often cystatin $\mathrm{C}$ yielded a different classification of kidney function compared to creatininebased equations for hospitalized patients receiving anticoagulation, since reclassification of kidney function could have important safety implications for these patients. We conducted a retrospective study of inpatients over a 1-year period who were prescribed anticoagulant medications during hospitalization and had both cystatin $\mathrm{C}$ and creatinine measured.

\section{METHODS}

\section{Study Design and Sample}

This was a retrospective cohort of patients admitted to the San Francisco VA Medical Center (SFVAMC) between February
2014 and February 2015 for whom serum cystatin C was ordered in addition to creatinine per provider discretion and who were either continued or started on prophylactic or therapeutic anticoagulation with non-VKA (vitamin K antagonist) anticoagulants, including direct oral anticoagulants (DOACs), low molecular weight heparins (LMWH), and fondaparinux. We did not include unfractionated heparin (UFH), as levels can be monitored using prothrombin time (PTT) and inpatient dosing does not rely on monitoring of kidney function. Providers at the SFVAMC have been able to order cystatin $\mathrm{C}$ without restriction since January 2013. The anticoagulation service began to use serum cystatin $\mathrm{C}$ in addition to serum creatinine measurements in 2014.

Between February 2014 and 2015, 368 inpatients received non-VKA, non-UFH anticoagulants; 141 of these patients had both serum creatinine and serum cystatin $\mathrm{C}$ drawn during the hospitalization. Patients were excluded if they did not have cystatin $\mathrm{C}$ and creatinine drawn on the same day. When multiple cystatin $\mathrm{C}$ and creatinine results were available for a hospitalization, only results from the first day were included. When a patient had multiple admissions, only the first admission was included. This yielded 75 patients in our study population.

The study received approval and waivers of informed consent and HIPAA authorization from the Institutional Review Board of the University of California, San Francisco.

\section{Measures}

Manual chart review was performed to abstract medical comorbidities, relevant laboratory data, medications, and indication for anticoagulation. We extracted sex, age, race (black, non-black, or unknown), and body mass index (BMI) by World Health Organization (WHO) classification; these are factors used in eGFR model calculations. We identified comorbid conditions that could affect creatinine or cystatin $\mathrm{C}$, including hypertension, diabetes, heart failure, active cancer within 1 year prior (solid tumor or hematologic malignancy), HIV infection, thyroid disease, and chronic liver disease (cirrhosis or persistently elevated liver function tests). Baseline hemoglobin and albumin levels at time of admission were also abstracted.

Creatinine and cystatin $\mathrm{C}$ were measured by routine laboratory assay on site. The creatinine assay was isotope dilution mass spectrometry (IDMS) standardized. Standardized cystatin C assays were performed on a Beckman Synchron DX600 analyzer with reagents produced by Gentian (Norway) and distributed by Beckman. ${ }^{18}$ Intra-assay coefficients of variation for cystatin $\mathrm{C}$, estimating within-run precision, ranged from 0.80 to $1.71 \%$ with mean serum concentrations between 0.96 and $2.95 \mathrm{mg} / \mathrm{L}$. Inter-assay coefficients of variation for cystatin C, estimating day-to-day precision, ranged from 2.76 to $3.37 \%$ with mean serum concentrations between 1.01 and $3.93 \mathrm{mg} / \mathrm{L}^{25}$ 
SFVAMC reports creatinine-based eGFR in $\mathrm{mL} / \mathrm{min} /$ $1.73 \mathrm{~m}^{2}$ by eGFR $\mathrm{MDRD}$, which was available to clinicians, but we also calculated eGFR by the eGFR $\mathrm{CG}_{\mathrm{C}}$ and eGFR $\mathrm{EPI}_{\mathrm{E}}$ equations for comparison. We calculated eGFR for cystatin $\mathrm{C}$ by the cystatin $\mathrm{C}$-specific CKD-EPI equation (eGFR $\left.\mathrm{ers}_{\mathrm{c}}\right)$. We did not use a combined creatinine-cystatin $\mathrm{C}$ equation since recent data suggest reduced accuracy of eGFR estimates using combined cystatin C-creatinine results when the individual estimates are not closely matched. ${ }^{30}$

We categorized eGFR based on each equation using two different classifications of kidney function: (1) standard eGFR stages by the Kidney Disease Improving Global Outcomes (KDIGO) guidelines and (2) drugdosing kidney classes (DDKC) based on large studies for the non-VKA anticoagulants.

Standard eGFR-based KDIGO stages are G1 (eGFR $\geq 90$ ), G2 (eGFR 60-89), G3a (eGFR 45-59), G3b (eGFR 30-44), stage 4 (eGFR 15-29), and G5 (eGFR $<15$ or dialysisdependent). ${ }^{31}$

The DDKC reflect cutoffs for dosing in pharmacologic studies of anticoagulants: class I (eGFR > 95), class II (eGFR 50-94), class III (eGFR 30-49), and class IV $(\mathrm{eGFR}<30){ }^{2,5}$ Class I represents patients who could potentially hyper-metabolize anticoagulant medications. ${ }^{32,33}$ Class II represents patients who should tolerate full-dose anticoagulation. Of note, a cutoff of $50 \mathrm{~mL} / \mathrm{min} / 1.73 \mathrm{~m}^{2}$ has been used most consistently across studies, but there are exceptions for different anticoagulant medications. ${ }^{5,34}$ Class III represents patients at risk for potential complications for whom dose reductions should be considered. Class IV represents patients with poor kidney function who may not be candidates for renally metabolized anticoagulants. We chose a cutoff of $30 \mathrm{~mL} / \mathrm{min} / 1.73 \mathrm{~m}^{2}$, as most studies excluded patients with $\mathrm{CrCl}<30 \mathrm{~mL} / \mathrm{min} / 1.73 \mathrm{~m}^{2}$; rivaroxaban is approved for $\mathrm{CrCl}$ between 15 and $49 \mathrm{~mL} / \mathrm{min} / 1.73 \mathrm{~m}^{2} .35,36$

\section{Outcomes}

There were three primary outcomes: the median of the withinpatient difference in eGFR, proportion of re-classification across KDIGO stages, and proportion of re-classification across drug-dosing classes.

Median differences in eGFR and interquartile ranges (IQR) were calculated based on eGFR using the cystatin C CKD-EPI equation minus eGFR using each of the three creatinine-based equations: eGFR $\mathrm{EPI}_{\mathrm{I}}$, eGFR $\mathrm{MDRD}_{\text {, and }} \mathrm{GFR}_{\mathrm{CG}}$. Re-classification of KDIGO stage was calculated as the proportion of patients for whom the cystatin $\mathrm{C}$ equation led to reclassification into a different KDIGO stage compared with each creatinine-based equation. Re-classification of DDKC was calculated as the proportion of patients for whom the cystatin $\mathrm{C}$ equation led to a re-classification into a different DDKC compared with each creatinine-based equation.

\section{Statistical Analysis}

To compare the distributions of eGFR calculated using each creatinine- or cystatin C-based equation, we plotted kernel density estimates of eGFR based on each equation using a Gaussian kernel and a smoothing bandwidth based on Silverman's rule. ${ }^{37}$

We calculated the median and interquartile range for eGFR based on the cystatin $\mathrm{C}$ equation and the three creatinine equations. Differences in the median eGFR by each creatinine-based equation compared to the cystatin $\mathrm{C}$ equation were assessed using two-sided paired sign tests.

Classifications of KDIGO stages of eGFR and drug-dosing kidney classes by the cystatin $\mathrm{C}$ equation were compared to corresponding classifications by the creatinine-based equations, and patients were grouped three subsets: worse, unchanged, or better kidney function when using cystatin $\mathrm{C}$ compared with the creatinine-based equation.

We described characteristics of the study sample across DDKC re-classification groups based on cystatin $\mathrm{C}$ versus creatinine using CKD-EPI equations. Although the MDRD and CKD-EPI equations for creatinine are the most commonly used in clinical practice, we chose the CKD-EPI equation because of its superior accuracy in comparison with MDRD. ${ }^{38}$ CKD-EPI is recommended by KDIGO, the National Institute of Diabetes and Digestive and Kidney Diseases, and the National Kidney Foundation. ${ }^{31,39,40}$ In addition, the CKDEPI cystatin $\mathrm{C}$ and creatinine equations are constructed by similar methods and thus are most comparable. We tested for differences in patient characteristics across the DDKC reclassification groups using chi-square tests for categorical variables and nonparametric Kruskal-Wallis tests for continuous variables. We also graphed the difference between cystatin- and creatinine-based eGFRs for each individual patient.

\section{RESULTS}

\section{Sample Characteristics}

Seventy-five inpatient veterans receiving non-VKA anticoagulants during hospitalization had both cystatin $\mathrm{C}$ and creatinine results available. Most were male, non-black, over 60 years old, and hypertensive (Table 1). Median age was 68 (IQR 65, 78), median body mass index was 27 (IQR 22, 32), median hemoglobin was 12.0 (IQR 10.0, 13.2), and median albumin was 3.2 (IQR 2.7, 3.6). About one third had diabetes. Thirteen (17\%) had cancer, including prostate (four patients), esophageal (three patients), and pancreatic (two patients). One patient each had renal cell, pelvic squamous cell, bladder, and lung cancer. One of the 13 was receiving chemotherapy at the time of the index hospitalization. Five had surgery for tumor resection during the index hospitalization. Indications for anticoagulant use were VTE prophylaxis (36\%), VTE treatment (39\%), and atrial fibrillation (25\%). Most patients received 
Table 1 Patient Characteristics by Reclassification of DDKC When Using Cystatin C Versus Creatinine (eGFR cys - eGFR $\left._{\text {EPI }}\right)$

\begin{tabular}{|c|c|c|c|c|c|}
\hline & \multirow[b]{2}{*}{$N$ (in group) } & \multirow{2}{*}{$\begin{array}{l}\text { DDKC better using cystatin C }(n=15) \\
n \text { (across row) }\end{array}$} & \multirow[t]{2}{*}{$\begin{array}{l}\text { No change } \\
(n=37)\end{array}$} & \multirow[t]{2}{*}{$\begin{array}{l}\text { DDKC worse using cystatin C } \\
(n=23)\end{array}$} & \multirow[b]{2}{*}{$p$ value } \\
\hline & & & & & \\
\hline \multicolumn{5}{|l|}{ Gender } & \multirow[t]{3}{*}{0.718} \\
\hline Female & $2(3)$ & $0(0)$ & $1(50)$ & $1(50)$ & \\
\hline Male & $73(97)$ & $15(21)$ & $36(49)$ & $22(30)$ & \\
\hline \multicolumn{5}{|l|}{ Age } & \multirow[t]{5}{*}{$<0.0005$} \\
\hline $40-59$ & $10(13)$ & $1(10)$ & $4(40)$ & $5(50)$ & \\
\hline $60-69$ & $31(41)$ & $14(45)$ & $11(35)$ & $6(19)$ & \\
\hline $70-79$ & $17(23)$ & $0(0)$ & $15(88)$ & $2(12)$ & \\
\hline $80-91$ & 17 (23) & $0(0)$ & $7(41)$ & $10(59)$ & \\
\hline \multicolumn{5}{|l|}{ Race } & \multirow[t]{4}{*}{0.891} \\
\hline Not black & $52(69)$ & $10(19)$ & $26(50)$ & $16(31)$ & \\
\hline Black & $6(8)$ & $2(33)$ & $3(50)$ & $1(17)$ & \\
\hline Unknown & $17(23)$ & $3(18)$ & $8(47)$ & $6(35)$ & \\
\hline \multicolumn{5}{|l|}{ Comorbidities } & \\
\hline Heart failure & & & & & \multirow[t]{3}{*}{0.549} \\
\hline No & $49(65)$ & $10(20)$ & $26(53)$ & $13(27)$ & \\
\hline Yes & $26(35)$ & $5(19)$ & $11(42)$ & $10(38)$ & \\
\hline \multicolumn{5}{|l|}{ Diabetes } & \multirow[t]{3}{*}{0.423} \\
\hline No & $51(68)$ & $9(18)$ & $24(47)$ & $18(35)$ & \\
\hline Yes & $24(32)$ & $6(25)$ & $13(54)$ & $5(21)$ & \\
\hline \multicolumn{5}{|l|}{ Hypertension } & \multirow[t]{3}{*}{0.266} \\
\hline No & $25(33)$ & $7(28)$ & $13(52)$ & $5(20)$ & \\
\hline Yes & $50(67)$ & $8(16)$ & $24(48)$ & $18(36)$ & \\
\hline \multicolumn{5}{|l|}{ Cancer } & \multirow[t]{3}{*}{0.03} \\
\hline No & $62(83)$ & $11(18)$ & $28(45)$ & $23(37)$ & \\
\hline Yes & 13 (17) & $4(31)$ & $9(69)$ & $0(0)$ & \\
\hline \multicolumn{5}{|l|}{ Thyroid disease } & \multirow[t]{3}{*}{0.584} \\
\hline No & $71(95)$ & $14(20)$ & $36(51)$ & $21(30)$ & \\
\hline Yes & $4(5)$ & $1(25)$ & $1(25)$ & $2(50)$ & \\
\hline \multicolumn{5}{|l|}{ Liver Disease } & \multirow[t]{3}{*}{0.263} \\
\hline No & $70(93)$ & $15(21)$ & $35(50)$ & $20(29)$ & \\
\hline Yes & $5(7)$ & $0(0)$ & $2(40)$ & $3(60)$ & \\
\hline \multicolumn{5}{|l|}{ BMI $\left(\mathrm{kg} / \mathrm{m}^{2}\right)$} & 0.852 \\
\hline Underweight $(<18.5)$ & $5(7)$ & $1(20)$ & $2(40)$ & $2(40)$ & \\
\hline Normal (18.5-25.0) & $27(36)$ & $5(19)$ & $13(48)$ & $9(33)$ & \\
\hline Overweight (25.0-30.0) & $17(23)$ & $3(18)$ & $11(65)$ & $3(18)$ & \\
\hline Obese $(>30.0)$ & $26(35)$ & $6(23)$ & $11(42)$ & $9(35)$ & \\
\hline Laboratory values & & & & & \\
\hline Hemoglobin $(\mathrm{g} / \mathrm{dL})$ & & & & & 0.605 \\
\hline$<12$ & 37 (49) & $6(16)$ & $18(49)$ & $13(35)$ & \\
\hline$\geq 12$ & $38(51)$ & $9(24)$ & $19(50)$ & $10(26)$ & \\
\hline Albumin (g/dL) & & & & & 0.178 \\
\hline$<3.2$ & $34(45)$ & $5(15)$ & $15(44)$ & $14(41)$ & \\
\hline$\geq 3.2$ & $41(55)$ & $10(24)$ & $22(54)$ & $9(22)$ & \\
\hline
\end{tabular}

DDKC: drug dosing kidney class, eGFRcys: cystatin-based glomerular filtration rate estimate, eGFRepi: estimate of glomerular filtration rate based on CKD-EPI equation, BMI: Body Mass Index (World Health Organization classifications)

low molecular weight heparin: $55(73 \%)$ had enoxaparin and 8 $(11 \%)$ received dalteparin. One patient $(1 \%)$ received fondaparinux. The remaining $11(15 \%)$ were on direct oral anticoagulants including dabigatran (one patient), apixaban (four patients), and rivaroxaban (six patients).

\section{eGFR by Cystatin C and Creatinine Equations}

Distributions of eGFR using each equation show that cystatin C-based estimates had a normal distribution peaking at a lower eGFR compared with the creatinine-based estimates (Fig. 1).

The median eGFR by cystatin $\mathrm{C}$ was $55.8 \mathrm{~mL} / \mathrm{min} / 1.73 \mathrm{~m}^{2}$ (IQR 43.0, 73.3), lower than the median eGFR by each of the three creatinine equations: $69.7 \mathrm{~mL} / \mathrm{min} / 1.73 \mathrm{~m}^{2}$ (IQR 50.7 , 86.8) for eGFR EPI $_{1}, 74.0 \mathrm{~mL} / \mathrm{min} / 1.73 \mathrm{~m}^{2}$ (IQR $56.0,112.0$ ) for eGFR $\mathrm{MDRD}_{\text {, and }} 84.5 \mathrm{~mL} / \mathrm{min} / 1.73 \mathrm{~m}^{2}$ (IQR $58.9,115.0$ ) for eGFR $\mathrm{CG}_{\mathrm{C}}$; all $p$ values $<0.01$. The median difference in eGFR using the cystatin $\mathrm{C}$ equation and the CKD-EPI equation for creatinine (eGFRcys - eGFR $\mathrm{EPI}_{\mathrm{I}}$ ) was $-7.1 \mathrm{~mL} / \mathrm{min} /$ $1.73 \mathrm{~m}^{2}$ (IQR - 17.2, 2.6), with an overall range of -67.9 to 38.8 (Fig. 2). The median difference between $\mathrm{eGFR}_{\mathrm{cys}}$ and eGFR ${ }_{\mathrm{MDRD}}$ was $-21.2 \mathrm{~mL} / \mathrm{min} / 1.73 \mathrm{~m}^{2}(\mathrm{IQR}-43.7,-8.1)$, and the largest median difference was between eGFR $\mathrm{R}_{\text {cys }}$ and $\mathrm{eGFR}_{\mathrm{CG}}$ : $-25.9 \mathrm{~mL} / \mathrm{min} / 1.73 \mathrm{~m}^{2}$ (IQR - 46.8, - 8.7) (see Appendix figures online).

\section{Reclassification of Drug-Dosing Kidney Class and KDIGO Stage}

Between 51 and $60 \%$ of patients were re-classified into different DDKC, and between 57 and $75 \%$ of patients were reclassified into different KDIGO kidney stages by cystatin $\mathrm{C}$ 


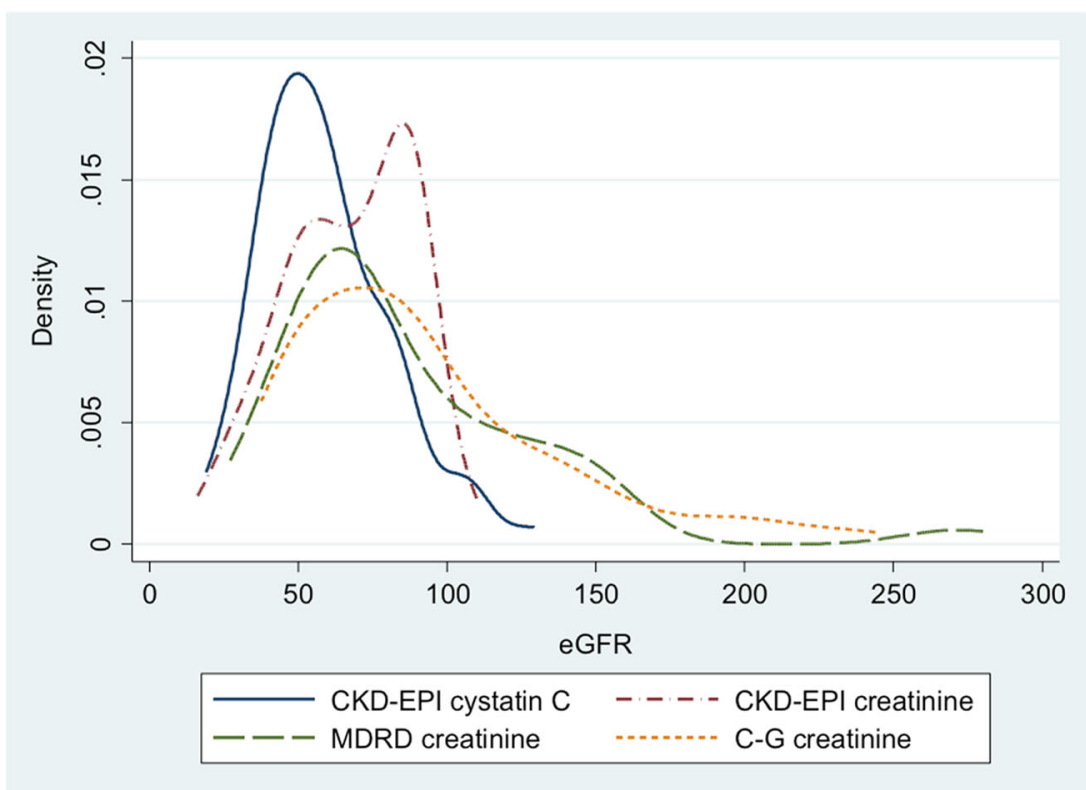

Figure 1 Smoothed kernel density plots of eGFR using each cystatin C- and creatinine-based equation. Distributions of eGFR (estimated glomerular filtration rate) using cystatin $C$ (eGFR ${ }_{\text {cys }}$, blue solid line), the creatinine-based CKD-EPI equation (eGFR EPI $_{\text {, }}$ red dotted-dashed line), the Modified Diet in Renal Disease (MDRD) equation (eGFR MDRD green dashed line), and the Cockcroft-Gault equation (eGFR ${ }_{C G}$, dotted yellow line). CKD-EPI: Chronic Kidney Disease Epidemiology Collaboration.

compared to creatinine-based equations (Fig. 3). Compared to $\mathrm{eGFR}_{\mathrm{EPI}}$, eGFR $\mathrm{eys}_{\text {cys }}$ led to reclassification of $31 \%$ of patients into a worse DDKC and $41 \%$ into a worse KDIGO kidney stage; these proportions were larger for the other creatininebased equations. Most reclassifications involved a change to a worse but adjacent DDKC or KDIGO stage (Fig. 4).

\section{Sensitivity Analysis Without Cancer Patients}

To evaluate whether the 13 patients with cancer influenced our findings, we repeated the analysis excluding them and found similar results. Median eGFR (mL/min/1.73 m²) was $51.4(\mathrm{IQR}$

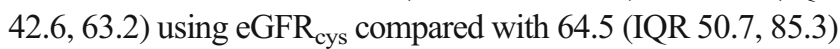

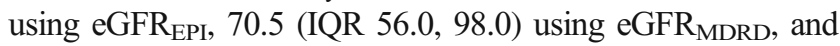

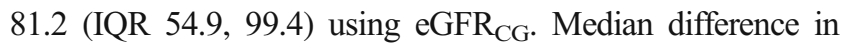

eGFR when using cystatin $\mathrm{C}$ was -7.6 (IQR - 21.3, 1.9) compared to eGFR $\mathrm{EPI},-20.7(\mathrm{IQR}-38.7,-6.7)$ compared to $\mathrm{eGFR}_{\mathrm{MDRD}}$, and -24.9 (IQR - 45.8, - 12.0) compared to eGFR $_{\mathrm{CG}}$. We found similar proportions of patients reclassified to different drug dosing kidney classes after we excluded the 13 patients with solid cancers. These proportions were $55 \%$ after excluding cancer versus $51 \%$ with the entire sample when we used the eGFR $\mathrm{EPI}_{\mathrm{E}}$ equation, 60 versus $60 \%$ with the $\mathrm{GFR}_{\mathrm{MDRD}}$ equation, and 61 versus $59 \%$ when using the $\mathrm{eGFR}_{\mathrm{CG}}$.

\section{DISCUSSION}

Accurate estimation of kidney function is important in patients receiving non-VKA anticoagulants who are at risk

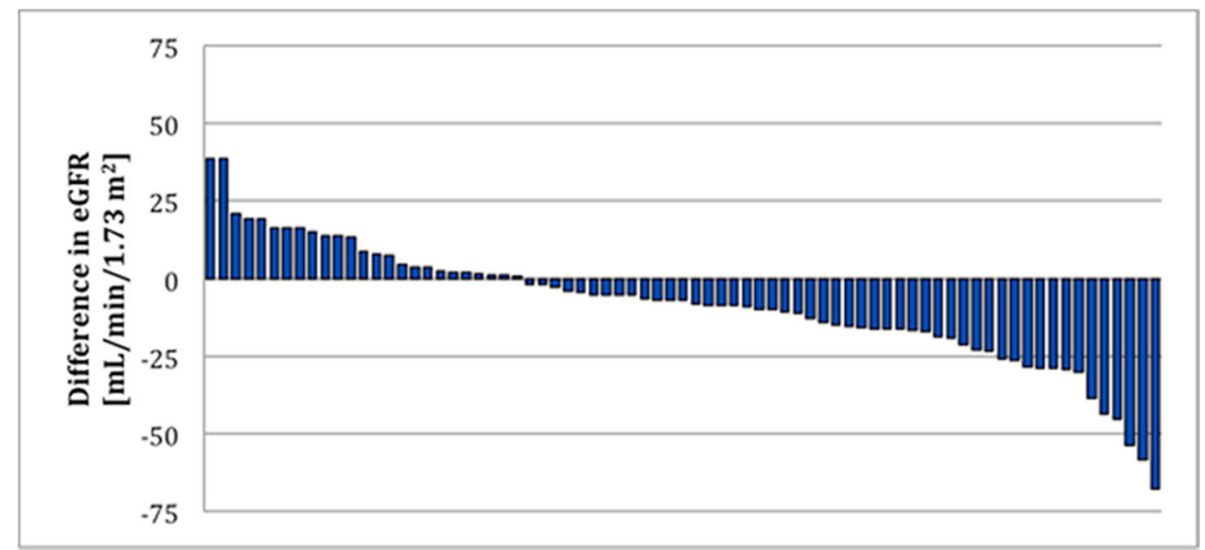

Figure 2 Magnitudes of individual patient differences between estimated eGFR using either cystatin C or creatinine-based eGFR (eGFRcys eGFREPI), $N=75$. Each bar represents an individual patient $(N=75)$; the magnitude of each bar represents the difference in estimated glomerular filtration rate (eGFR) based on cystatin C and eGFR based on creatinine, using the CKD-EPI equations (eGFR cys - eGFR $_{\text {EPI }}$ ). Negative values represent patients whose cystatin C-based eGFR estimates are lower than the corresponding creatinine estimates. CKD-EPI: Chronic Kidney Disease Epidemiology Collaboration. 


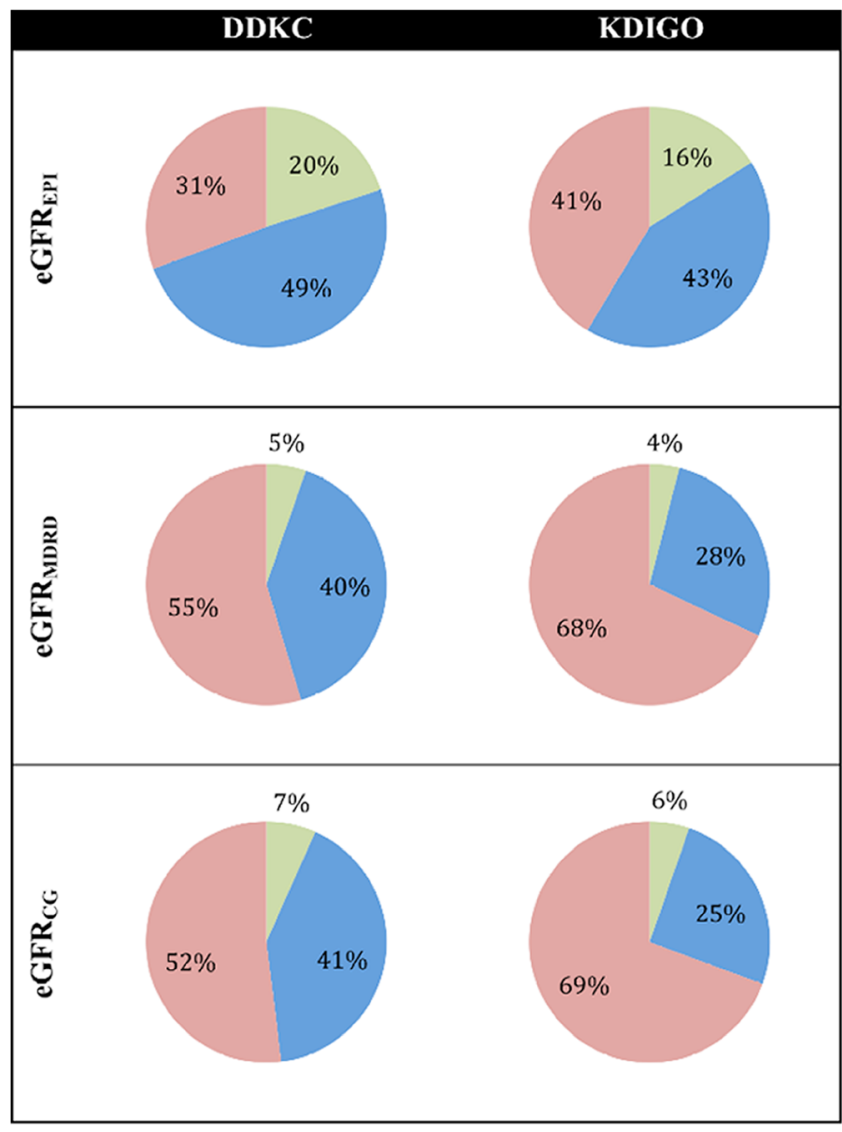

Figure 3 Proportions of patients re-classified into different drug dosing kidney classes (DDKC) and KDIGOstages based on cystatin $\mathrm{C}$ versus each creatinine-based equation. Proportions of patients reclassified to different drug dosing kidney classes (DDKC, left column) and KDIGO kidney stages (right column) using eGFR compared to eGFR EPI $_{\text {(top row), }}$ eGFR $_{\text {MDRD }}$ (middle row), or eGFR $_{\text {CG }}$ (bottom row). Green sections represent reclassification into better (higher eGFR) categories using eGFR cys $_{\text {; }}$ blue sections represent no change; and red sections show reclassification into worse categories. KDIGO: Kidney Disease: Improving Global Outcomes; eGFR: estimated glomerular filtration rate; eGFR cys $_{\text {: }}$ eGFR based on CKD-EPI equation for cystatin C; eGFR $_{\text {EPI }}$ eGFR based on CKD-EPI equation for creatinine; eGFR $_{\text {MDRD: }}$ eGFR based on the Modified Diet in Renal Disease equation; eGFR $_{\text {CG }}$ GFR based on the Cockcroft-Gault equation; CKD-EPI: Chronic Kidney Disease Epidemiology Collaboration.

for bleeding and thrombotic complications from inappropriate dosing. We found discordance between eGFR using cystatin $\mathrm{C}$ compared to all creatinine-based estimates $\left(\mathrm{eGFR}_{\mathrm{EPI}}\right.$, eGFR $\mathrm{MDRD}_{\mathrm{MD}}$, and $\left.\mathrm{eGFR}_{\mathrm{CG}}\right)$ in a cohort of hospitalized patients receiving anticoagulation. Between 51 and $60 \%$ of patients were re-classified into a different drugdosing kidney class when cystatin $\mathrm{C}$ was used, and in most cases, cystatin $\mathrm{C}$ led to lower estimates of kidney function.

We also noted variation in eGFR estimates based on the different creatinine-based equations. The CKD-EPI equation was most aligned with estimates based on cystatin $C$; however, there was still a $-7 \mathrm{~mL} / \mathrm{min} / 1.73 \mathrm{~m}^{2}$ difference in median eGFR and a wide range of individual differences (Fig. 2). Of the creatinine-based estimates, $\mathrm{eGFR}_{\mathrm{EPI}}$ had the lowest proportion of reclassifications; however, $31 \%$ of patients were still reclassified into a worse drug dosing category by cystatin C. Cystatin C led to reclassification of over $50 \%$ of patients into a worse drug dosing class compared to the MDRD and CG creatinine-based estimates.

Our findings remained unchanged in sensitivity analyses excluding cancer patients. Progression of malignancy leading to high cell turnover may lead to elevated cystatin $\mathrm{C} .{ }^{41}$ However, none of the patients in this study had cancer with classically high cell turnover, and only one was receiving chemotherapy.

Our study of hospitalized patients showed greater discordance than prior studies comparing cystatin $\mathrm{C}$ and creatinine as markers of kidney function, which have generally been done in clinical cohorts of volunteers with better health status than their peers. ${ }^{18,21,23}$ For instance, a meta-analysis of 11 studies comparing eGFR by creatinine and cystatin C found that among over 90,000 participants in the general population with a creatinine-based eGFR using CKD-EPI of 60 to $89 \mathrm{~mL} /$ $\min / 1.73 \mathrm{~m}^{2}, 14 \%$ were reclassified to a cystatin C-based eGFR of less than $60 \mathrm{~mL} / \mathrm{min} / 1.73 \mathrm{~m}^{2}{ }^{42}$ In our cohort, 17 of the 40 patients with eGFR $\mathrm{EPI} 60-89 \mathrm{~mL} / \mathrm{min} / 1.73 \mathrm{~m}^{2}(43 \%)$ were reclassified into lower categories.

Our hospitalized population included patients with acute illness and many comorbid conditions; the mean albumin of $3.2 \mathrm{~g} / \mathrm{dL}$ was lower than average albumin levels of approximately $4.0 \mathrm{~g} / \mathrm{dL}$ observed in cohorts of elders in other large studies of cystatin C. ${ }^{43-45}$ The medical complexity and poor health status of this population may contribute to the discordance we observed.

The primary limitations of this study are the retrospective design and the sample size. Since the use of cystatin $\mathrm{C}$ is relatively new at our institution, this group of patients may have been perceived at higher risk for an inaccurate GFR estimate based on creatinine and therefore may not be reflective of all inpatients on anticoagulation. However, this is the group of patients in whom this clinical issue is most relevant.

The study sample was limited in demographic scope, as the VA inpatient population is predominantly male and elderly. Relevant comorbidities, including active malignancy with high cell turnover and uncontrolled thyroid disease, were not sufficiently represented for their effects on estimates of kidney function to be assessed. In addition, we did not assess for acute kidney injury (AKI); however, the recommended clinical practice for patients with known significant AKI is to use UFH rather than other non-VKA anticoagulation.

We did not assess whether anticoagulant doses were actually adjusted throughout the hospitalization or whether adjustments were based on cystatin $\mathrm{C}$ results. There are no prior studies assessing cystatin $\mathrm{C}$ as a tool to refine anticoagulant dosing, although cystatin $\mathrm{C}$ has shown promise in guiding dosing of other high-risk medications including metformin in outpatients with diabetes and hospitalized inpatients receiving vancomycin. ${ }^{25,46}$ Due to our limited sample size, we could not address relevant patient-level outcomes such as bleeding, venous thromboembolism, and ischemic or embolic stroke. 


\begin{tabular}{|c|c|c|c|c|c|c|c|c|c|c|c|c|c|}
\hline \multicolumn{7}{|c|}{ Drug-Dosing Kidney Class } & \multicolumn{7}{|c|}{ KDIGO Stage } \\
\hline & \multicolumn{6}{|c|}{ eGFR $_{\text {cys }}$} & & \multicolumn{6}{|c|}{ eGFR $_{\text {cys }}$} \\
\hline \multirow{6}{*}{ 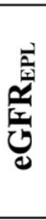 } & & I & II & III & IV & Total & \multirow{7}{*}{ 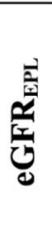 } & & & $3 \mathbf{a}$ & $3 \mathbf{b}$ & 4 & Total \\
\hline & I & 1 & 2 & 1 & 0 & 4 & & 1 & & 2 & 2 & 0 & 8 \\
\hline & II & 4 & 31 & 17 & 1 & 53 & & 2 & 0 & 11 & 5 & 1 & 40 \\
\hline & III & 0 & 6 & 5 & 2 & 13 & & $3 \mathbf{a}$ & & 7 & 6 & 0 & 14 \\
\hline & IV & 0 & 1 & 4 & 0 & 5 & & $\mathbf{3 b}$ & & 3 & 3 & 2 & 8 \\
\hline & Total & 5 & 40 & 27 & 3 & 75 & & 4 & & 0 & 4 & 0 & 5 \\
\hline & & & & & & & & To & 4 & 23 & 20 & 3 & 75 \\
\hline
\end{tabular}

\begin{tabular}{|c|c|c|c|c|c|c|}
\hline & & & & $\mathbf{R}_{\mathrm{cy}}$ & & \\
\hline & & I & II & III & IV & Total \\
\hline$\overline{\underline{\underline{\mu}}}$ & I & 5 & 17 & 3 & 0 & 25 \\
\hline$\alpha^{\bar{z}}$ & II & 0 & 20 & 18 & 1 & 39 \\
\hline 昰 & III & 0 & 3 & 5 & 2 & 10 \\
\hline 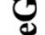 & IV & 0 & 0 & 1 & 0 & 1 \\
\hline & Total & 5 & 40 & 27 & 3 & 75 \\
\hline
\end{tabular}

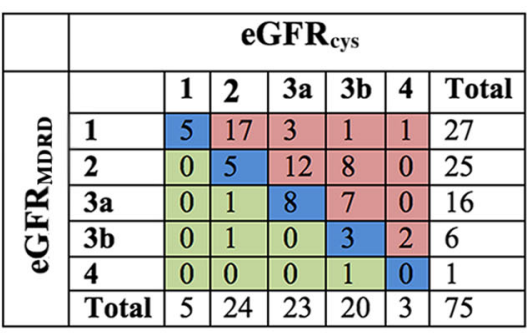

\begin{tabular}{|l|l|l|l|l|l|l|}
\hline & \multicolumn{7}{|c|}{ EGFR $_{\text {cys }}$} \\
\hline \multirow{3}{*}{$\underset{\mho}{*}$} & & I & II & III & IV & Total \\
\cline { 2 - 7 } & I & 4 & 17 & 4 & 0 & 25 \\
\cline { 2 - 7 } & II & 1 & 19 & 15 & 2 & 37 \\
\cline { 2 - 7 } & III & 0 & 4 & 8 & 1 & 13 \\
\cline { 2 - 7 } & IV & 0 & 0 & 0 & 0 & 0 \\
\cline { 2 - 7 } & Total & 5 & 40 & 27 & 3 & 75 \\
\hline
\end{tabular}

\begin{tabular}{|l|l|l|l|l|l|l|l|}
\hline & \multicolumn{7}{|c|}{ EGFR $_{\text {cys }}$} \\
\hline \multirow{4}{*}{$\underset{\mathscr{c}}{ }$} & & $\mathbf{1}$ & $\mathbf{2}$ & $\mathbf{3 a}$ & $\mathbf{3 b}$ & $\mathbf{4}$ & Total \\
\cline { 2 - 8 } & $\mathbf{1}$ & 5 & 15 & 8 & 4 & 0 & 32 \\
\cline { 2 - 8 } & $\mathbf{2}$ & 0 & 6 & 10 & 7 & 1 & 24 \\
\cline { 2 - 7 } & $\mathbf{3 a}$ & 0 & 3 & 4 & 5 & 2 & 14 \\
\cline { 2 - 7 } & $\mathbf{3 b}$ & 0 & 0 & 1 & 4 & 0 & 5 \\
\cline { 2 - 7 } & $\mathbf{4}$ & 0 & 0 & 0 & 0 & 0 & 0 \\
\cline { 2 - 7 } & Total & 5 & 24 & 23 & 20 & 3 & 75 \\
\hline
\end{tabular}

Figure 4 Numbers of patients re-classified into different KDIGO stages or drug dosing class based on cystatin C versus each creatinine-based equation. Numbers of individual patients in each drug dosing kidney class (left) and each KDIGO stage (right), based on eGFR cys $_{\text {and }}$ on each creatinine-based eGFR equation. Green cells represent reclassification into better (higher eGFR) categories; blue cells represent no change; and red sections show reclassification into worse (lower eGFR) categories. KDIGO: Kidney Disease: Improving Global Outcomes; eGFR estimated

glomerular filtration rate; eGFR cys $_{\text {eGFR based on CKD-EPI equation for cystatin C; eGFR }}$ EPI: eGFR based on CKD-EPI equation for creatinine; eGFR MDRD $_{\text {: eGFR based on the Modified Diet in Renal Disease equation; eGFR }}$ : $_{\text {: }}$ eGFR based on the Cockcroft-Gault equation; CKD-EPI: Chronic Kidney Disease Epidemiology Collaboration.

Larger studies are needed to evaluate the impact of using cystatin $\mathrm{C}$ to guide anticoagulant dosing on clinical outcomes and patient safety. We focused on a population of inpatients on anticoagulation, but our findings also highlight need for improved guidance in dosing of other medications such as antibiotics, chemotherapy, and contrast dye.

\section{CONCLUSION}

We found substantial discordance in cystatin C-based estimated kidney function compared to creatinine-based estimates in our study of hospitalized patients receiving non-VKA anticoagulation. Further studies are needed to determine whether routine testing of cystatin $\mathrm{C}$ for hospitalized patients decreases adverse outcomes, and to identify groups of patients for whom cystatin $\mathrm{C}$ testing might be most useful. In the meantime, clinicians should consider using cystatin $\mathrm{C}$ to help determine appropriate doses of renally metabolized anticoagulants for hospitalized patients.
Acknowledgments: This material is the result of work supported with resources and the use of facilities at the San Francisco Veteran Affairs Medical Center.

Corresponding Author: Erika Leemann Price, MD, MPH; Department of MedicineUniversity of California, San Francisco (UCSF), San Francisco, CA, USA (e-mail: Erika.price@ucsf.edu).

Compliance with Ethical Standards

The study received approval and waivers of informed consent and HIPAA authorization from the Institutional Review Board of the University of California, San Francisco.

Conflict of Interest: The authors declare that they have no conflicts of interest.

\section{REFERENCES}

1. Parks AL, Fang MC. Anticoagulation in older adults with multimorbidity. Clin Geriatr Med. 2016;32(2):331-46.

2. Fontana $\mathbf{P}$, Robert-Ebadi H, Bounameaux $\mathbf{H}$, Boehlen $\mathbf{F}$, Righini $\mathbf{M}$. Direct oral anticoagulants: a guide for daily practice. Swiss Med Wkly. 2016;146:w14286.

3. Wang Y, Bajorek B. New oral anticoagulants in practice: pharmacological and practical considerations. Am J Cardiovasc Drugs. 2014;14(3):175-89. 
4. Del-Carpio Munoz F, Gharacholou SM, Munger TM, et al. Metaanalysis of renal function on the safety and efficacy of novel oral anticoagulants for atrial fibrillation. Am J Cardiol. 2016;117(1):69-75.

5. Hart RG, Eikelboom JW, Ingram AJ, Herzog CA. Anticoagulants in atrial fibrillation patients with chronic kidney disease. Nat Rev Nephrol. 2012;8(10):569-78.

6. Robert-Ebadi H, Righini M. Anticoagulation in the elderly. Pharmaceuticals. 2010;3(12):3543-69.

7. Odden MC, Tager IB, Gansevoort RT, et al. Age and cystatin C in healthy adults: a collaborative study. Nephrol Dial Transplant. 2010;25(2):463-69.

8. White RH, Zhou H, Gage BF. Effect of age on the incidence of venous thromboembolism after major surgery. J Thromb Haemost. 2004;2(8):1327-33.

9. Ballew SH, Chen Y, Daya NR, et al. Frailty, kidney function, and polypharmacy: the atherosclerosis risk in communities (ARIC) study. Am J Kidney Dis. 2017;69(2):228-36.

10. Delanaye $\mathbf{P}$, Mariat $\mathbf{C}$. The applicability of eGFR equations to different populations. Nat Rev Nephrol. 2013;9(9):513-22.

11. Levey AS, Inker LA. Assessment of Glomerular Filtration Rate in Health and Disease: A State of the Art Review. Clin Pharmacol Ther. 2017;102(3):405-419.

12. Cockcroft DW, Gault MH. Prediction of creatinine clearance from serum creatinine. Nephron. 1976;16(1):31-41.

13. Sunder S, Jayaraman R, Mahapatra HS, et al. Estimation of renal function in the intensive care unit: the covert concepts brought to light. J Intensive Care. 2014;2(1):31.

14. Perrone RD, Madias NE, Levey AS. Serum creatinine as an index of renal function: new insights into old concepts. Clin Chem. 1992;38(10):1933-1953.

15. Matzke GR, Aronoff GR, Atkinson AJ, Jr., et al. Drug dosing consideration in patients with acute and chronic kidney disease-a clinical update from Kidney Disease: Improving Global Outcomes (KDIGO). Kidney Int. 2011;80(11):1122-37.

16. Rocco MV, Berns JS. KDOQI clinical practice guideline for diabetes and CKD: 2012 update. Am J Kidney Dis. 2012;60(5):850-86.

17. Baxmann AC, Ahmed MS, Marques NC, et al. Influence of muscle mass and physical activity on serum and urinary creatinine and serum cystatin C. Clin J Am Soc Nephrol. 2008;3(2):348-54.

18. Inker LA, Schmid $\mathbf{C H}$, Tighiouart $\mathbf{H}$, et al. Estimating glomerular filtration rate from serum creatinine and cystatin C. N Engl J Med. 2012;367(1):20-29.

19. Modig S, Lannering C, Östgren CJ, Mölstad S, Midlöv P. The assessment of renal function in relation to the use of drugs in elderly in nursing homes; a cohort study. BMC Geriatr. 2011;11:1

20. Peralta CA, Shlipak MG, Judd S, et al. Detection of chronic kidney disease with creatinine, cystatin $\mathrm{C}$, and urine albumin-to-creatinine ratio and association with progression to end-stage renal disease and mortality. JAMA. 2011;305(15):1545-1552.

21. Shlipak MG, Mattes MD, Peralta CA. Update on cystatin C: incorporation into clinical practice. Am J Kidney Dis. 2013;62(3):595-603.

22. Weinert LS, Camargo EG, Soares AA, Silveiro SP. Glomerular filtration rate estimation: performance of serum cystatin $\mathrm{C}$-based prediction equations. Clin Chem Lab Med. 2011;49(11):1761-71.

23. Zhang $\mathbf{Z}, \mathbf{L u} \mathbf{B}$, Sheng $\mathbf{X}$, Jin $\mathbf{N}$. Cystatin $\mathrm{C}$ in prediction of acute kidney injury: a systemic review and meta-analysis. Am J Kidney Dis. 2011;58(3):356-65.

24. Odden MC, Scherzer R, Bacchetti P, et al. Cystatin C level as a marker of kidney function in human immunodeficiency virus infection: the FRAM study. Arch Intern Med. 2007;167(20):2213-2219.
25. Tuot DS, Scherzer R, Leong H, Hung AM, Grunfeld C, Shlipak MG. Use of cystatin $\mathrm{C}$ to inform metformin eligibility among adult veterans with diabetes. J Clin Transl Endocrinol. 2016;3:1-6.

26. Villa P, Jimenez M, Soriano MC, Manzanares J, Casasnovas P. Serum cystatin $\mathrm{C}$ concentration as a marker of acute renal dysfunction in critically ill patients. Crit Care. 2005;9(2) R139-143.

27. Herget-Rosenthal S, Marggraf G, Husing J, et al. Early detection of acute renal failure by serum cystatin C. Kidney Int. 2004;66(3) 1115-1122.

28. Segarra A, de la Torre J, Ramos N, et al. Assessing glomerular filtration rate in hospitalized patients: a comparison between CKD-EPI and four cystatin C-based equations. Clin J Am Soc Nephrol. 2011;6(10) 24112420 .

29. Chertow GM, Burdick E, Honour M, Bonventre JV, Bates DW. Acute kidney injury, mortality, length of stay, and costs in hospitalized patients. J Am Soc Nephrol. 2005; 16(11) 3365-3370.

30. Bjork J, Grubb A, Larsson A, et al. Accuracy of GFR estimating equations combining standardized cystatin $\mathrm{C}$ and creatinine assays: a cross-sectional study in Sweden. Clin Chem Lab Med. 2015;53(3):403-414.

31. Kidney Disease: Improving Global Outcomes (KDIGO) CKD Work Group. KDIGO 2012 clinical practice guideline for the evaluation and management of chronic kidney disease. Kidney Int Suppl. 2013;3(5):1-150.

32. Guirguis E, Brown D, Grace Y, Patel D, Henningfield S. Establishing edoxabans role in anticoagulation. J Pharm Pract. 2016;29(3):228-38.

33. Trikha R, Kowey PR. Practical considerations for the nonvitamin $\mathrm{K}$ antagonist oral anticoagulants. Cardiology. 2017;136(2):115-24.

34. Patel MR, Mahaffey $\mathbf{K W}$, Garg $\mathbf{J}$, et al. Rivaroxaban versus warfarin in nonvalvular atrial fibrillation. N Engl J Med. 2011;365(10):883-91.

35. Granger CB, Alexander JH, McMurray JJV, et al. Apixaban versus warfarin in patients with atrial fibrillation. N Engl J Med. 2011;365(11):981-92.

36. Connolly SJ, Eikelboom J, Joyner C, et al. Apixaban in patients with atrial fibrillation. N Engl J Med. 2011;364(9):806-817.

37. Silverman BW. Density Estimation for Statistics and Data Analysis. London: Chapman \& Hall; 1986.

38. Inker LA, Shaffi K, Levey AS. Estimating glomerular filtration rate using the chronic kidney disease-epidemiology collaboration creatinine equation: better risk predictions. Circ Heart Fail. 2012;5(3):303-306.

39. National Institute of Diabetes and Digestive and Kidney Diseases. Estimating glomerular filtration rate. https://www.niddk.nih.gov/healthinformation/communication-programs/nkdep/laboratory-evaluation/ glomerular-filtration-rate/estimating. Accessed 26 December 2017.

40. GFR calculator - The National Kidney Foundation. https://www.kidney. org/professionals/kdoqi/gfr_calculator. Accessed December 26, 2017.

41. Kos J, Stabuc B, Cimerman N, Brunner N. Serum cystatin C, a new marker of glomerular filtration rate, is increased during malignant progression. Clin Chem. 1998;44(12):2556-2557.

42. Shlipak MG, Matsushita K, Arnlov J, et al. Cystatin C versus creatinine in determining risk based on kidney function. N Engl $\mathrm{J}$ Med. 2013;369(10):932-943.

43. Keller C, Katz R, Sarnak MJ, et al. Inflammatory biomarkers and decline in kidney function in the elderly: the Cardiovascular Health Study. Nephrol Dial Transplant. 2010;25(1):119-24

44. Lang J, Scherzer R, Tien PC, et al. Serum albumin and kidney function decline in HIV-infected women. Am J Kidney Dis. 2014;64(4):584-91.

45. Lang J, Scherzer R, Weekley CC, Tien PC, Grunfeld C, Shlipak MG. Serum albumin and short-term risk for mortality and cardiovascular disease among HIV-infected veterans. AIDS. 2013;27(8):1339-43.

46. Frazee EN, Rule AD, Herrmann SM, et al. Serum cystatin C predicts vancomycin trough levels better than serum creatinine in hospitalized patients: a cohort study. Crit Care. 2014;18(3):R110. 